\title{
Entropy and its mathematical properties: consequences for thermodynamics
}

\author{
Martina Costa Reis - Caio César Ferreira Florindo • \\ Adalberto Bono Maurizio Sacchi Bassi
}

Received: 3 December 2014/ Accepted: 29 January 2015/Published online: 24 February 2015

(C) Springer International Publishing 2015

\begin{abstract}
In this work, a comprehensive meaning for entropy is provided on the basis of foundations of information theory and statistical thermodynamics. For this purpose, the close relation between missing information and entropy is presented by emphasizing their probabilistic nature. Furthermore, the physical implications of the mathematical properties of the entropy function are exploited using the elementary notions of differential and integral calculus. Particularly, it is evidenced that the usual thermodynamic inequalities found in many textbooks of physical chemistry are direct consequences of the concavity of entropy. The aim of this work is to show that many concepts presented in textbooks of physical chemistry can be obtained in a simple and mathematically clear way.
\end{abstract}

Keywords Entropy · Statistical thermodynamics . Information theory $\cdot$ Concave functions

\section{List of symbols}

$\begin{array}{ll}S & \text { Entropy } \\ Q & \text { Heat } \\ T & \text { Temperature } \\ U & \text { Internal energy } \\ V & \text { Volume } \\ p & \text { Pressure } \\ n & \text { Amount of substance } \\ N & \text { Number of particles } \\ N_{\mathrm{A}} & \text { Avogadro constant } \\ t & \text { Time } \\ \psi & \text { Information }\end{array}$

M. C. Reis $(\varangle)$ - C. C. F. Florindo - A. B. M. S. Bassi Department of Physical Chemistry, Institute of Chemistry, University of Campinas, UNICAMP, Campinas, Brazil e-mail: marreis@iqm.unicamp.br

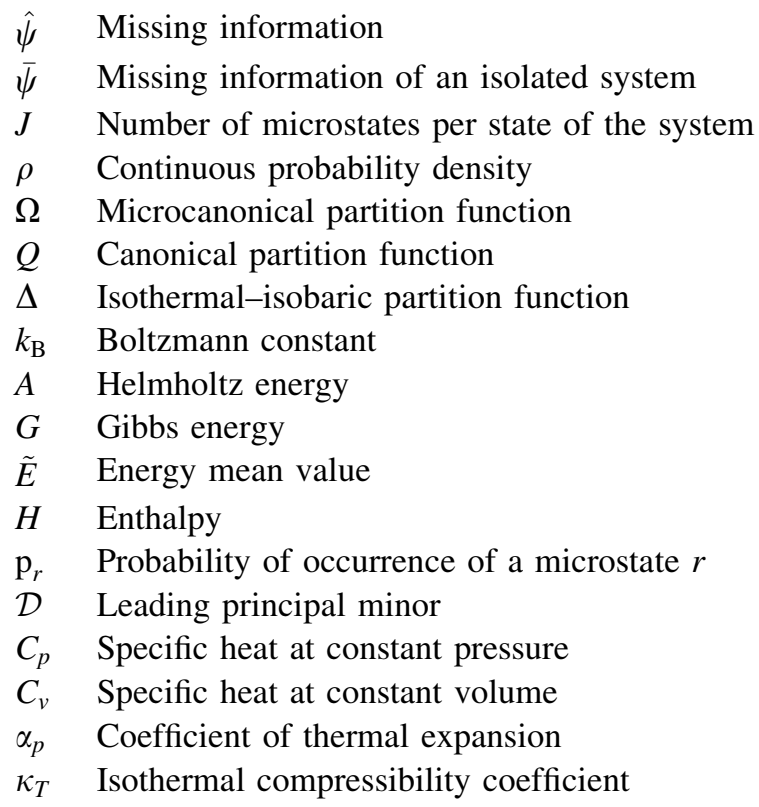

\section{Introduction}

Thermodynamics has occupied a central role in physical chemistry as its foundations have supported the understanding of many physicochemical phenomena. In electrochemistry, for instance, thermodynamic fundamentals have been used to develop important phenomenological equations, as well as theoretical models for electric double layer [1, 2]. In surface chemistry [3], Gibbs' thermodynamic approach has been systematically used to study interfacial phenomena, such as the adsorption of chemical species on a particular substrate and stability of colloidal dispersions. Moreover, in materials science [4], thermodynamic concepts have been employed to construct phase 
diagrams and to foresee the material behaviors of systems under different physicochemical conditions.

However, while the number of reports on thermodynamic applications have extraordinarily increased in the last decades, a well-motivated discussion about the most elementary thermodynamic concepts has been neglected in the current chemical literature. As a matter of fact, the few works that provide a mathematically consistent discussion about basic thermodynamic concepts are concentrated in journals of the areas of mathematics, physics, and mechanical engineering [5-7]. The mathematical framework used by these manuscripts is frequently uncommon to most of the chemists, who are secluded from the most recent conceptual advances in thermodynamics. As a consequence, usually no new thermodynamic approach is taught in undergraduate and graduate courses, and the discussion in many textbooks of physical chemistry is restricted to the conventional formalism of equilibrium thermodynamics.

In view of this context, many basic ideas of thermodynamics are axiomatically introduced and sometimes in a mathematically incoherent form. The discussion that follows in several textbooks of physical chemistry is then in many aspects intuitive, so that the relation between mathematical properties and thermodynamic consequences is overlooked. A typical example of such a situation is the presentation of the entropy and the content of the second law of thermodynamics for which several physical interpretations and mathematical formulations can be found.

In this work, a well-motivated physical interpretation for entropy is presented on the basis of foundations of information theory. For this purpose, the probabilistic nature of the microstates of a system is emphasized by using statistical thermodynamic concepts inherent to chemistry students. Furthermore, the mathematical properties of entropy function are exploited by employing only elementary concepts of differential and integral calculus. Particularly, by postulating the existence of the entropy $S$ as a function of internal energy $U$ and volume $V$, it is shown that thermodynamic inequalities commonly posed in textbooks of physical chemistry can be obtained as direct consequences of the concavity of entropy function with respect to the variables $U$ and $V$.

Despite the simple mathematical methodology employed in this manuscript, many concepts presented here are still unknown to most chemistry students. Thus, the content of this work could be useful in courses of advanced thermodynamics and for those interested in a mathematically coherent description of thermodynamics.

\section{Historical perspective of entropy}

The literature $[8,9]$ is full of different formulations for the second law of thermodynamics, as well as different physical interpretations for entropy. One of the most known interpretations relates entropy to the molecular order/disorder of a system. However, since this interpretation rests on merely qualitative ideas, and no general quantitative description of molecular order/disorder has been conceived, a number of works have appeared, questioning the relation between entropy and molecular order/ disorder [10, 11]. As a matter of fact, since the mid-1950s several works, e.g., [12-14] have presented a new approach, which associates entropy to the concept of missing information, such as in the mathematical information theory. Even so, there are still many chemists, physicists, and engineers who prefer to deal with entropy as the molecular disorder of a system.

In 1865, when Clausius [15] introduced the concept of entropy, even Clausius himself was not very satisfied by his definition. Based on Carnot's work [16] about heat engines, Clausius proposed an expression,

$\frac{Q_{\text {boiler }}}{T_{\text {high }}}=\frac{\left|Q_{\text {cooler }}\right|}{T_{\text {low }}}$,

which allowed him to describe the heat transfers $Q$ in a Carnot cycle. According to Clausius, it is clear from Eq. (1) that during a Carnot cycle, it is not the heat transfers that are kept unchanged, but rather a quantity, called entropy, given by the ratio of heat and temperature $T$. In fact, the word entropy (from Greek, transformation) was intentionally used by Clausius because he believed that energy and entropy were closely related, although the units of these quantities are quite different.

The earliest associations of entropy with molecular disorder were made from Maxwell's and Boltzmann's works $[17,18]$. In the mid-1870s, Maxwell and Boltzmann worked at the same time on the foundations of kinetic theory of gases. Nevertheless, it was Boltzmann who first provided the statistical interpretation of entropy with his famous formula, which relates entropy to the total number of microstates of a system whose energy, volume, and number of particles are well defined. Actually, Boltzmann proposed a theorem, where an H-quantity always decreases and attains its minimum value at equilibrium as a consequence of molecular collisions and other feasible assumptions. Boltzmann also noticed that his H-quantity behaved in a similar way to the quantity $S$ introduced by Clausius, but by no means stated that the increase of entropy implies the increase of molecular disorder of a system. In fact, this conclusion appeared just after consolidation of the atomistic view of matter and consequent application of Boltzmann's ideas in simple situations, such as the expansion of an ideal gas and mixing of two different gases in a closed vessel. In these examples, the increase of entropy was related to the fact that particles spread all over the volume of the vessel, resulting in greater molecular disorder. 
Until the mid-1940s, the relation between entropy and molecular disorder prevailed in the literature. However, with the divulgation of mathematical information theory proposed by Shannon [19] in 1948 and subsequent publication of Brillouin's book [12], and Jaynes' manuscript [13], the association of entropy with molecular disorder has been changed. With new thermodynamic concepts available in the literature and the development of modern mathematical theories, the discussion of the mathematical foundations of thermodynamics gained prominence in the areas of physics and mathematics in the late 1960s and 1970s. Examples of important publications in this subject are Truesdell's and Gile's works [20, 21], which discuss comprehensively the mathematical foundations of classical thermodynamics based on the inequalities

$\left.\frac{\partial U}{\partial T}\right|_{V}>0,\left.\quad \frac{\partial p}{\partial V}\right|_{T}<0$,

where $p$ is the pressure. The approach used in these works is founded on the idea that the existence of entropy function should not be a priori assumed, but rather must be proved. There, as well as in other works [22, 23], the proof of the concavity of entropy function and its physical implications are demonstrated by considering an isolated system, which is subdivided into two subsystems in thermal equilibrium with each other.

On the other hand, if one follows Gibbs' thought, the existence of entropy function is ensured as a primitive concept, and one should exploit the consequences therefrom. From this point of view, one exploits the concavity of entropy function in relation to certain variables to obtain the two inequalities presented in Eq. (2), as well as other important thermodynamic consequences in a mathematically clear way.

\section{Entropy and information theory}

Suppose that a macroscopic system has pressure $p$, amount of substance $n$, and temperature $T$ well defined. Although the knowledge of these parameters may be enough to define the state of the system, it tells nothing about the positions and velocities of the constituent particles of the system. By supposing that it contains $N$ particles, where $N$ is given by $N=N_{\mathrm{A}} n$ and $N_{\mathrm{A}}$ is the Avogadro constant $\left(N_{\mathrm{A}}=6.02214179(30) \times 10^{23} \mathrm{~mol}^{-1}\right)$, one should know the values of $6 \mathrm{~N}$ variables - three Cartesian components of the position vector, and three Cartesian components of the velocity vector for each particle-to describe fully the microscopic configuration of the system. Thus, to characterize the microstate at a given instant $t$, one should know the values of a number of variables of the order of magnitude of $10^{23}$, whereas to determine the macroscopic state of the system at that time $t$, it may suffice to know the values of three variables, e.g., $p, T$, and $n$.

Of course, for any macroscopic system, the determination of the values of about $10^{23}$ variables is unachievable. Nevertheless, one may associate to each state of the system the number of all its compatible microstates. For this purpose, one initially considers a thermodynamic process, whereby a state (1) is transformed into the state (2) through a continuous sequence of states. Then, one defines the number of microstates $J$ per state of the system, such that $J$ is dimensionless. Furthermore, since every state corresponds to at least one microstate, it follows that $J \geq 1$. Accordingly, because of the great number of particles in a macroscopic system, one should expect an enormous number of microstates in the equilibrium state.

By recalling the foundations of information theory, ${ }^{1}$ let $\psi$ be the sufficient quantity of information to define the microstate in which the system is located. Of course, this microstate must be compatible with the macroscopic restrictions imposed on the system, e.g., temperature, pressure, and mass. If more than one microstate is compatible with such restrictions, there will be a certain quantity of missing information (uncertainty) $\hat{\psi}$ with respect to the knowledge of the state. Note yet that the variables required to specify the state of a thermodynamic system change according to the kind of process to which the system is submitted. For example, the macroscopic restrictions used to define $\psi$ and $\hat{\psi}$ for a given state of an isolated system are not the same as those used to define $\psi$ and $\hat{\psi}$ for a state of a closed system submitted to an isothermal process and vice versa. Similarly, different conditions apply to systems submitted to other kinds of processes.

Once the quantities of information and missing information have been defined, a relation between $\hat{\psi}$ and $J$ may be proposed. Thus, for a macroscopic isolated system, postulate that (i) all microstates compatible with a state of the system are equally probable [24]; (ii) $\hat{\psi}$ must be a nonnegative strictly increasing continuous function of $J$, so that the larger the number of microstates, the greater is the amount of missing information; (iii) if at any instant $t$, an observer was capable of knowing all macroscopic properties of the system, as well as the exact position and velocity of the constituent particles, the observer would unambiguously know the microstate of the system at that instant. Therefore, $\hat{\psi}(1)=0$, where $J=1$; and (iv) for $j$ independent systems, the total amount of missing information is the sum of the missing information of $j$ independent systems. In turn, the total number of microstates is given by

\footnotetext{
${ }^{1}$ An accessible presentation of the foundations of information theory may be found in the references $[10,11]$.
} 
the product of the number of microstates of $j$ independent systems, viz.

$\hat{\psi}=\sum_{i=1}^{j} \hat{\psi}_{i}, \quad J=\prod_{i=1}^{j} J_{i}$

Hence, based on the Shannon entropy formula [19], one supposes that the expression for the missing information of an isolated system $\bar{\psi}$ must be of the form of

$\bar{\psi}=\beta \log _{a}(J)$,

where $\beta=\beta^{(a)} \ln (a)$ is an information measure unit determined by the base $a$ of the logarithm. For example, when $a=2$, then $\bar{\psi}$ and $\beta$ are given in terms of the basic indissoluble information unit (bit). Thus, it follows that the missing information is determined by binary choices

$\bar{\psi}=\beta^{(2)} \ln (J), \quad \beta^{(2)}=\frac{\beta}{\ln (2)}$.

Remark 1 By using the change-of-base formula for logarithms, Eq. (4) may be alternatively written as

$\bar{\psi}=\beta^{(a)} \ln (J)$

Equation (5) is similar to the Boltzmann entropy formula that relates the entropy $S$ to the number of microstates of an isolated system,

$S=k_{\mathrm{B}} \ln (J)$,

such that $k_{\mathrm{B}}$ is the Boltzmann constant $\left(k_{\mathrm{B}}=1.3806504(24) \times 10^{-23} \mathrm{~J} \mathrm{~K}^{-1}\right)$. Thus, note that the ratio of expressions (6) and (5) results in

$S=\alpha \bar{\psi}, \quad \alpha=\frac{k_{\mathrm{B}}}{\beta} \ln (a)$,

where $\alpha$ is a proportionality constant that transforms a given information measure unity into $\mathrm{J} \mathrm{K}^{-1}$. Hence, one concludes that, for an isolated system, entropy is proportional to missing information.

Remark 2 In statistical thermodynamics, Boltzmann entropy formula is also given in terms of the microcanonical partition function $\Omega(N, V, E)$,

$S=k_{\mathrm{B}} \ln (\Omega)$,

where the partition function $\Omega$ expresses the number of microstates available to the isolated system in a given energy.

Moreover, there are other entropy-like functions that maintain a proportionality relation with their corresponding missing information quantities. For instance, for a system represented by a canonical ensemble, the corresponding missing information is proportional to $-A / T$, where $A$ is the Helmholtz energy. Likewise, for a system represented by an isothermal-isobaric ensemble, the associated missing information is proportional to $-G / T$, where $G$ is the Gibbs energy. Thus, in analogy to expression (7), one writes the formulas

$-\frac{A}{T}=k_{\mathrm{B}} \ln (Q) \quad$ and, $\quad-\frac{G}{T}=k_{\mathrm{B}} \ln (\Delta)$,

where $Q(N, V, T)$ denotes the canonical partition function, and $\Delta(N, T, p)$ stands for the isothermal-isobaric partition function $[25,26] .^{2}$

\section{The case of non-uniform probability distribution}

Consider a macroscopic isolated system presenting $J$ microstates. As previously stated, the quantity $S$ is related to the number of microstates of the system by the expression

$S=k_{\mathrm{B}} \ln (J)$.

Because the microstates $J$ of this system have equal probability of occurrence $p=1 / J$, Eq. (6) becomes

$$
\begin{aligned}
S & =-k_{\mathrm{B}} \ln \left(\frac{1}{J}\right)=-k_{\mathrm{B}} J\left[\frac{1}{J} \ln \left(\frac{1}{J}\right)\right]=-k_{\mathrm{B}} J[\mathrm{p} \ln (\mathrm{p})] \\
& =-k_{\mathrm{B}} \sum_{r=1}^{J} \mathrm{p} \ln (\mathrm{p}) .
\end{aligned}
$$

Actually, Eq. (9) is a particular case of the general equation

$$
S=-k_{\mathrm{B}} \sum_{r=1}^{J} \mathrm{p}_{r} \ln \left(\mathrm{p}_{r}\right),
$$

where Eq. (10) is subject to the normalization condition $\sum_{r=1}^{J} \mathrm{p}_{r}=1$. This equation is useful to evaluate the entropy of systems modeled by canonical and isothermalisobaric ensembles [25, 26].

One can also propose an expression analogous to Eq. (10), where $S$ is given in terms of a continuous probability density function $\rho(r)$ on an interval $[0, J]$. Hence, one has

$S=-k_{\mathrm{B}} \int_{0}^{J} \rho(r) \ln (\rho(r)) \mathrm{d} r$,

where Eq. (11) is subject to the condition $\int_{0}^{J} \rho(r) \mathrm{d} r=1$. As a matter of fact, whenever the restriction of microstates with equal probability of occurrence is imposed, Eq. (11) reduces to Eq. (6).

\footnotetext{
2 The reader interested in the deduction of expressions presented in Eq. (8) should consult pages 37-45 of reference [25], and pages 12-18 of reference [26].
} 


\section{Mathematical properties of the entropy function}

Consider a closed system represented by a canonical ensemble, such as that described in [24]. Admit also that each state of this system only has two possible microstates, so that $\mathrm{p}_{1}+\mathrm{p}_{2}=1$. Then, by focusing on a particular state of this system, it follows from Eq. (10) that

$S=-k_{\mathrm{B}}\left[\mathrm{p}_{1} \ln \left(\mathrm{p}_{1}\right)+\left(1-\mathrm{p}_{1}\right) \ln \left(1-\mathrm{p}_{1}\right)\right]$,

whose graph representation is provided in Fig. 1.

According to Fig. 1, (i) $S$ has only one global maximum at $\mathrm{p}_{1}=1 / 2$, that is, when both microstates have equal probabilities of occurrence, (ii) $S$ attains its minimum value, when one knows precisely which microstate is associated with a given state of the system, and (iii) the function $S\left(\mathrm{p}_{1}\right)$ is concave. Mathematically, this implies that

(a) The first derivative of $S\left(\mathrm{p}_{1}\right)$ always decreases as $\mathrm{p}_{1}$ increases;

(b) The second derivative of $S\left(\mathrm{p}_{1}\right)$ is always negative;

(c) The local maximum is always a global maximum;

(d) The Hessian matrix of $S$ is negative definite [27]. This property is of particular interest since the expressions shown in Eq. (2) and many other thermodynamic inequalities are directly deduced from the Hessian matrix of $S(U, V)$. In the next section, a simple example of the exploitation of this property will be presented.

Remark 3 A function $f(x)$ is concave on an interval $[a, b]$, if for any two $x_{1}$ and $x_{2}$ in $[a, b]$, the value of the concave function $f(x)$ at the point $\left(x_{1}+x_{2}\right) / 2$ is always larger than or equal to $\left[f\left(x_{1}\right)+f\left(x_{2}\right)\right] / 2$,

$$
\frac{f\left(x_{1}\right)+f\left(x_{2}\right)}{2} \leq f\left(\frac{x_{1}+x_{2}}{2}\right) \text {. }
$$

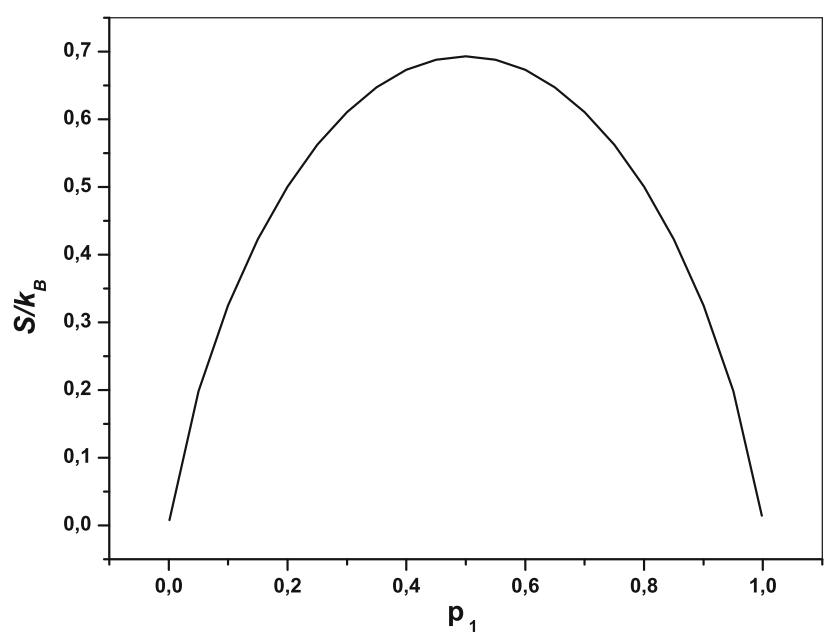

Fig. 1 The ratio $S / k_{\mathrm{B}}$ versus $\mathrm{p}_{1}$, with $\mathrm{p}_{1}=1-\mathrm{p}_{2}$
Examples of concave functions are $f(x)=\log (x)$, $f(x)=-x \log (x), f(x)=-x^{2}$ and $f(x)=\sqrt{x}$ [28].

Clearly, the above example does not correspond to the physical reality of an ordinary macroscopic system. However, one can extend the foregoing conclusions without loss of generality for usual macroscopic closed systems, whose states are related to an enormous number of microstates $J$ with different probabilities $\mathrm{p}_{r}$. Accordingly, for a system with a finite number of microstates, $S$ attains its maximum value $^{3}$ only when all microstates are equally probable, that is, the maximum value of $S$ is given by Eq. (6). To see this, consider the generalization of the inequality presented in Remark 3 for the case of $n$ points in $[a, b]$,

$\frac{f\left(x_{1}\right)+f\left(x_{2}\right)+\cdots+f\left(x_{n}\right)}{n} \leq f\left(\frac{x_{1}+x_{2}+\cdots+x_{n}}{n}\right)$.

By applying the above inequality to Eq. (10), one has

$-k_{\mathrm{B}} \frac{\sum_{r=1}^{J} \mathrm{p}_{r} \ln \left(\mathrm{p}_{r}\right)}{J} \leq-k_{\mathrm{B}} \frac{\sum_{r=1}^{J} \mathrm{p}_{r}}{J} \ln \left(\frac{\sum_{r=1}^{J} \mathrm{p}_{r}}{J}\right)$.

Then, by multiplying Eq. (13) by $J$ and recalling that $\sum_{r=1}^{J} \mathrm{p}_{r}=1$, one obtains

$-k_{\mathrm{B}} \sum_{r=1}^{J} \mathrm{p}_{r} \ln \left(\mathrm{p}_{r}\right) \leq-k_{\mathrm{B}} \ln \left(\frac{1}{J}\right)$,

or yet

$-k_{\mathrm{B}} \sum_{r=1}^{J} \mathrm{p}_{r} \ln \left(\mathrm{p}_{r}\right) \leq k_{\mathrm{B}} \ln (J)$,

where the equality holds if and only if $\mathrm{p}_{r}=\mathrm{p}=1 / J$. This is an important result as it shows that a canonical ensemble becomes a microcanonical one. For closed systems with a number of constituent particles of the order of magnitude of $10^{23}$, the probability of the system to have an energy very different from the energy mean value $\tilde{E}$ is nearly null. In this case, the only microstates that contribute significantly to the sum $\sum_{r=1}^{J} \mathrm{p}_{r} \ln \left(\mathrm{p}_{r}\right)$ are those with energy mean value $\tilde{E}$ [26]. Indeed, such microstates $J$ are those which present a probability of occurrence $\mathrm{p}_{r}$ equal to $\mathrm{p}=1 / \mathrm{J}$, that is, they are exactly the same microstates of a microcanonical ensemble. Under such circumstances, the macroscopic properties of a canonical ensemble, e.g., entropy $S$ are identical to those of a microcanonical ensemble,

\footnotetext{
${ }^{3}$ By using the method of Lagrange multipliers, it is possible to demonstrate that $S$ attains its maximum value only when all microstates have equal probability of occurrence. This demonstration may be found on pages 331-333 of reference [10].
} 
and one says the canonical ensemble degenerated into a microcanonical one [24, 25].

Hitherto, the concavity of entropy function with respect to the probability of occurrence of a particular microstate $r$ of a closed system has been discussed. Nevertheless, for thermodynamic purposes, it would be interesting to consider the concavity of entropy function $S$ in relation to certain macroscopic variables. Thus, postulate that $S$ is a concave function of both internal energy $U$ and volume $V$. The motivation of this postulate rests on the results that will be discussed in the next section.

\section{Thermodynamic consequences of the concavity of entropy function}

By following Gibbs' approach, the entropy function is a primitive concept and thermodynamic properties of a macroscopic system with a fixed amount of substance are determined by the relations that exist between its volume $V$, pressure $p$, temperature $T$, internal energy $U$, and entropy $S$. This may be done by the well-known thermodynamic relation,

$$
\mathrm{d} S=\frac{1}{T} \mathrm{~d} U+\frac{p}{T} \mathrm{~d} V=\left(\left.\frac{1}{T} \frac{\partial U}{\partial V}\right|_{T}+\frac{p}{T}\right) \mathrm{d} V+\left.\frac{1}{T} \frac{\partial U}{\partial T}\right|_{V} \mathrm{~d} T,
$$

where $U$ is a function $U(V, T)$ and

$\left.\frac{\partial S}{\partial U}\right|_{V}=\frac{1}{T},\left.\quad \frac{\partial S}{\partial V}\right|_{U}=\frac{p}{T}$,

or yet,

$$
\left.\frac{\partial S}{\partial V}\right|_{T}=\left.\frac{1}{T} \frac{\partial U}{\partial V}\right|_{T}+\frac{p}{T},\left.\quad \frac{\partial S}{\partial T}\right|_{V}=\left.\frac{1}{T} \frac{\partial U}{\partial T}\right|_{V} .
$$

Here, it is opportune to emphasize the convenience of setting $S(U, V)$. Gibbs, in his works [29] about geometrical representation of the thermodynamic properties of heterogeneous substances, chose to work with the variables volume $V$, internal energy $U$, and entropy $S$. On the other hand, J. Thomson, brother of Lord Kelvin, preferred to work with the variables volume $V$, pressure $p$, and temperature $T$. However, while the set proposed by J. Thomson is completely determined by a proper differentiation of the set $(V, U, S)$, the latter cannot be determined from the former. This occurs because while $S(U, V)$ is a fundamental equation, $p(V, T)$ is an equation of state.

By regarding the dependence of $S$ in terms of $(U, V)$, one uses the Taylor's formula with remainder [27] to represent $S(U, V)$ as a polynomial at any point $\left(U^{o}, V^{o}\right)$ of the thermodynamic phase space,

$$
\begin{aligned}
S(U, V)= & S\left(U^{o}, V^{o}\right)+\left(U-U^{o}\right) \frac{\partial S}{\partial U}\left(U^{o}, V^{o}\right) \\
& +\left(V-V^{o}\right) \frac{\partial S}{\partial V}\left(U^{o}, V^{o}\right) \\
& +\frac{1}{2}\left(U-U^{o}\right)^{2} \frac{\partial^{2} S}{\partial U^{2}}\left(U^{o}, V^{o}\right) \\
& +\left(U-U^{o}\right)\left(V-V^{o}\right) \frac{\partial^{2} S}{\partial U \partial V}\left(U^{o}, V^{o}\right) \\
& +\frac{1}{2}\left(V-V^{o}\right)^{2} \frac{\partial^{2} S}{\partial V^{2}}\left(U^{o}, V^{o}\right)+R(2),
\end{aligned}
$$

where $R(2)$ is the remainder of the Taylor's series. The second-order derivatives of the above polynomial are the elements of the Hessian matrix of $S(U, V)$. Thus, from the property $(d)$ of concave functions, the Hessian matrix of $S(U, V)$,

$\left[\begin{array}{cc}\frac{\partial^{2} S}{\partial U^{2}} & \frac{\partial^{2} S}{\partial U \partial V} \\ \frac{\partial^{2} S}{\partial V \partial U} & \frac{\partial^{2} S}{\partial V^{2}}\end{array}\right]$

must be negative definite. From matrix (19), one exploits the thermodynamic consequences of the concavity of entropy function in relation to $U$ and $V$. For this purpose, one needs to consider the definiteness of this matrix,

$\mathcal{D}_{1}=\frac{\partial^{2} S}{\partial U^{2}}<0, \quad \mathcal{D}_{2}=\left|\begin{array}{cc}\frac{\partial^{2} S}{\partial U^{2}} & \frac{\partial^{2} S}{\partial U \partial V} \\ \frac{\partial^{2} S}{\partial V \partial U} & \frac{\partial^{2} S}{\partial V^{2}}\end{array}\right|>0$,

where $\mathcal{D}_{1}$ and $\mathcal{D}_{2}$ stand for the leading principal minors of first and second orders, respectively, and the signs of the leading principal minors are determined according to the rule $(-1)^{k}$, with $k$ being the order of the leading principal minor.

Initially, one looks at $\mathcal{D}_{1}$. As $\left.\frac{\partial S}{\partial U}\right|_{V}=\frac{1}{T}$, this results in $\left.\frac{\partial^{2} S}{\partial U^{2}}\right|_{V}=-\left.\frac{1}{T^{2}} \frac{\partial U}{\partial T}\right|_{V} ^{-1}$, and, therefore,

$\left.\frac{\partial U}{\partial T}\right|_{V}>0$,

where expression (21) is a known thermodynamic inequality (see Eq. (2) 1 ). At this point, it is worthwhile to observe that the concavity of $S(U, V)$ implies that the partial derivative shown in expression (21) is invertible.

The thermodynamic consequences of $\mathcal{D}_{2}$ follow from 
$\left.\left.\frac{\partial^{2} S}{\partial U^{2}}\right|_{V} \frac{\partial^{2} S}{\partial V^{2}}\right|_{U}-\frac{\partial^{2} S}{\partial V \partial U}\left(\frac{\partial^{2} S}{\partial U \partial V}\right)>0$.

Accordingly, from expression $(16)_{2}$ one determines

$\left.\frac{\partial^{2} S}{\partial V^{2}}\right|_{U}=\left.\frac{1}{T} \frac{\partial p}{\partial V}\right|_{U}-\left.\frac{p}{T^{2}} \frac{\partial T}{\partial V}\right|_{U}$.

But, if one considers $p$ as a function of $V$ and $T$, there remains

$\left.\frac{\partial p}{\partial V}\right|_{U}=\left.\frac{\partial p}{\partial V}\right|_{T}+\left.\left.\frac{\partial p}{\partial T}\right|_{V} \frac{\partial T}{\partial V}\right|_{U}$,

whence

$\left.\frac{\partial^{2} S}{\partial V^{2}}\right|_{U}=\left.\frac{1}{T} \frac{\partial p}{\partial V}\right|_{T}+\left.\left.\frac{1}{T} \frac{\partial p}{\partial T}\right|_{V} \frac{\partial T}{\partial V}\right|_{U}-\left.\frac{p}{T^{2}} \frac{\partial T}{\partial V}\right|_{U}$.

This expression can be further simplified by considering $\left.\frac{\partial p}{\partial T}\right|_{V}=\left.\frac{1}{T} \frac{\partial U}{\partial V}\right|_{T}+\frac{p}{T}$, which comes from Eq. (17) $)_{1}$ and $\left.\frac{\partial S}{\partial V}\right|_{T}=\left.\frac{\partial p}{\partial T}\right|_{V}$. This last expression is one of Maxwell's relations that can be obtained from the differential $\mathrm{d} A=-p \mathrm{~d} V-S \mathrm{~d} T$. Thus, one has that

$\left.\frac{\partial^{2} S}{\partial V^{2}}\right|_{U}=\left.\frac{1}{T} \frac{\partial p}{\partial V}\right|_{T}+\left.\left.\frac{1}{T^{2}} \frac{\partial T}{\partial V}\right|_{U} \frac{\partial U}{\partial V}\right|_{T}$,

which may be rewritten as

$\left.\frac{\partial^{2} S}{\partial V^{2}}\right|_{U}=\left.\frac{1}{T} \frac{\partial p}{\partial V}\right|_{T}-\left.\frac{1}{T^{2}} \frac{\partial T}{\partial U}\right|_{V}\left(\left.\frac{\partial U}{\partial V}\right|_{T}\right)^{2}$,

where $\left.\frac{\partial T}{\partial V}\right|_{U}=-\left.\left.\frac{\partial T}{\partial U}\right|_{V} \frac{\partial U}{\partial V}\right|_{T}$ was used. Therefore, inequality (22) reduces to

$\left.\frac{\partial^{2} S}{\partial U^{2}}\right|_{V}\left[\left.\frac{1}{T} \frac{\partial p}{\partial V}\right|_{T}-\left.\frac{1}{T^{2}} \frac{\partial T}{\partial U}\right|_{V}\left(\left.\frac{\partial U}{\partial V}\right|_{T}\right)^{2}\right]-\frac{\partial^{2} S}{\partial V \partial U}\left(\frac{\partial^{2} S}{\partial U \partial V}\right)>0$,

where the expression within the brackets must be negative in view of inequality $(20)_{1}$. To ensure this, one recalls inequality (21). Then, it follows that

$\left.\frac{\partial p}{\partial V}\right|_{T}<0$,

which is another important thermodynamic inequality [see Eq. $\left.(2)_{2}\right]$.

Moreover, further thermodynamic inequalities can be obtained from expressions (21) and (29). With inequality (29), and $\left.\frac{\partial A}{\partial V}\right|_{T}=-p$, one obtains

$\left.\frac{\partial^{2} A}{\partial V^{2}}\right|_{T}>0$.
In turn, by using inequality (21), Eq. (17) 2 , and $\left.\frac{\partial A}{\partial T}\right|_{V}=-S$, one has

$\left.\frac{\partial^{2} A}{\partial T^{2}}\right|_{V}<0$

Similarly, one can deduce

$\left.\frac{\partial^{2} G}{\partial p^{2}}\right|_{T}<0, \quad$ and $\left.\quad \frac{\partial^{2} G}{\partial T^{2}}\right|_{p}<0$,

which also are thermodynamic constraints. Such inequalities imply that the Helmholtz energy $A$ is a concave function of $T$, but a convex function of $V$, as well as the Gibbs energy $G$ is a concave function of both $T$ and $p$.

Another thermodynamic constraint of particular importance restricts the values of the specific heat at constant pressure $C_{p}=\left.\frac{\partial H}{\partial T}\right|_{p}$ and the specific heat at constant volume $C_{v}=\left.\frac{\partial U}{\partial T}\right|_{V}$, where $H$ is the enthalpy. From Eq. (15), one deduces

$C_{p}-C_{v}=-\left.\left.T \frac{\partial p}{\partial V}\right|_{T} \frac{\partial V}{\partial T}\right|_{p} ^{2}$,

which, with the help of expression (29), leads to

$C_{p}>C_{v}$.

Furthermore, by using the definitions of the coefficients of thermal expansion $\alpha_{p}=\left.\frac{1}{V} \frac{\partial V}{\partial T}\right|_{p}$ and isothermal compressibility $\kappa_{T}=-\left.\frac{1}{V} \frac{\partial V}{\partial p}\right|_{T}$, one writes the relation between $C_{p}$ and $C_{v}$ as

$C_{p}=C_{v}+\frac{\alpha_{p}^{2}}{\kappa_{T}} T V$

Thus, from expressions (21) and (29), it follows immediately that

$\alpha_{p}^{2}<\frac{1}{T V} C_{p} \kappa_{T}$.

\section{Conclusion}

In the late twentieth century, several attempts were made to give a clearer and well-motivated definition for the entropy and the second law of thermodynamics. In this work, it was discussed how mathematical properties of the entropy function led to important conclusions in thermodynamics. For this purpose, Gibbs' approach was used, so that the existence of an entropy function dependent of the internal 
energy and volume was taken for granted. Indeed, it was shown that entropy is closely related to missing information. Then, it was presented in a straightforward way that the concavity of entropy implies

$\left.\frac{\partial U}{\partial T}\right|_{V}>0,\left.\quad \frac{\partial p}{\partial V}\right|_{T}<0, \quad C_{p}>C_{v}$,

as well as other usual thermodynamic inequalities found in textbooks of physical chemistry.

Acknowledgments M. C. Reis and C. C. F. Florindo acknowledge the São Paulo Research Foundation (Grant 2013/20872-2) and CAPES (Foundation for the Improvement of Higher Education Personnel) for their funding.

\section{References}

1. Bockris JOM, Reddy AKN (1998) Modern electrochemistry 1: ionics. Plenum Press, New York

2. Bockris JOM, Reddy AKN, Gamboa-Aldeco ME (1998) Modern electrochemistry 2A: fundamentals of electrodics. Plenum Press, New York

3. Hiemenz PC, Rajagopalan R (1997) Principles of colloids and surface chemistry. Marcel Dekker, New York

4. DeHoff R (2006) Thermodynamics in materials science. CRC Press, Boca Raton

5. Truesdell CA (1984) Rational thermodynamics. Springer, Berlin

6. Hutter K (1977) Acta Mech 27:1-54

7. Coleman BD, Owen DR (1974) Arch Ration Mech Anal 54:1-104

8. Müller I (2007) A history of thermodynamics. The doctrine of energy and entropy. Springer, Berlin

9. Truesdell CA (1980) The tragicomical history of thermodynamics. Springer, Berlin
10. Ben-Naim A (2008) A farewell to entropy: statistical thermodynamics based on information. World Scientific, Toh Tuck Link

11. Ben-Naim A (2007) Entropy demystified. The second law reduced to plain common sense. World Scientific, Toh Tuck Link

12. Brillouin L (1956) Science and information theory. Academic Press, New York

13. Jaynes ET (1957) Phys Rev 106:620-630

14. Liboff RL (1974) J Stat Phys 11:343-357

15. Clausius RJE (1865) Ann Phys Chem 125:353-400

16. Carnot NLS (1824) Refléxions sur la puissance motrice du feu et sur les machines propres à développer cette puissance. Chez Bachelier, Paris

17. Maxwell JC (1965) The scientific papers of James Clerk Maxwell. Dover, New York

18. Boltzmann LE (1872) Wien Ber 66:275-370

19. Shannon CE (1948) Bell Syst Tech J. doi:10.1002/j.1538-7305. 1948.tb01338.x

20. Truesdell CA, Baratha S (1977) The concepts and logic of classical thermodynamics as a theory of heat engines. Springer, New York

21. Giles R (1964) Mathematical foundations of thermodynamics. Pergamon, Oxford

22. Callen HB (1985) Thermodynamics and an introduction to thermostatics. Wiley, New York

23. Galgani L, Scotti A (1970) Pure Appl Chem. doi:10.1351/ pac197022030229

24. Tolman RC (1938) The principles of statistical mechanics. Dover, Oxford

25. McQuarrie DA (1976) Statistical mechanics. Harper \& Row, New York

26. Hill TL (1960) An introduction to statistical thermodynamics. Addison-Wesley, Reading

27. Apostol TM (1967) Calculus. Wiley, New York

28. Webster R (1995) Convexity. Oxford Press, Oxford

29. Gibbs JW (1906) The scientific papers of J. Williard Gibbs. Thermodynamics. Longmans-Green, Madison 